\section{Diagrammatic representation of lacrimal disease}

valuable after surgery, with syringing, to assess ostium function. ${ }^{9,10}$ Findings are recorded in the notes using a combination of diagrams and text.

We aimed to develop a clear diagrammatic record of lacrimal findings, before and after surgery, which could be used by ophthalmologists of all grades within the clinic.

\title{
Methods
}

We looked at existing methods for strabismus, ${ }^{11,12}$ cornea $^{13,14}$ and for lacrimal disease. ${ }^{15,16}$ We developed the system described within a specialist lacrimal clinic at a teaching hospital. A standard proforma (on one A4 page) was designed (Fig. 1) which included space for the history (in text) and examination findings (diagrams with minimum text). Ocular surface and lid causes of hypersecretion are recorded in the external eye examination. Diagrammatic representation of the lacrimal system findings is subdivided as follows:

\section{Periocular, eyelids and puncta}

Patients presenting with watering eyes are common in ophthalmic practice and may be seen by different ophthalmologists with a range of lacrimal experience. It is important to have a standard approach for recording lacrimal disease - which first excludes the presence of staphylococcal lid disease and secondary corneal abnormalities then concentrates on assessing the lacrimal drainage system.

Conway, ${ }^{1}$ in a survey of ophthalmologists, showed that the lacrimal assessment was inconsistent and recommended a standard approach. Unfortunately, there are many tests used to assess the lacrimal system. ${ }^{2-5}$ Some of the dye tests are now rarely used as they have a low sensitivity. ${ }^{1,6}$ A systematic approach using more than one clinical diagnostic test, including dacryocystography and/or nuclear lacrimal scintillography, is necessary to differentiate the causes of epiphora from hypersecretion and locate the most likely site of stenosis. ${ }^{7,8}$

Our approach includes examination of the periocular area, eyelids, external eye, tear meniscus, dye retention test, syringing and endonasal examination. The last excludes nasal causes of epiphora and identifies anatomical variations which influence surgery. It is also
Medial canthal masses, fistulae and eyelid malposition are recorded on a line drawing of the eyelids with the medial canthal tendon outlined. Symbols for the puncta are used, e.g. normal puncta are shown as small solid circles (Table 1). Lid malposition is superimposed on the eyelid outline and medial canthal masses recorded in relation to the medial canthal tendon.

\section{Tear meniscus and dye retention test}

The vertical height of the tear meniscus is estimated at the slit lamp before instillation of drops and is recorded as a simple line drawing. The fluorescein dye retention test (FDRT) is then performed; this test is also called the fluorescein disappearance test (FDT). It is recorded 3 and $5 \mathrm{~min}$ after instillation of guttae fluorescein $2 \%$ into the conjunctival fornix. The dye test is positive when there is residual fluorescein in the tear lake and is graded +1 to +3 or any asymmetry noted. No reduction in fluorescein is represented by +3 whereas +1 represents minimal residual dye. Zero represents complete rapid clearance. 
Date.

History

Symptoms

$\mathrm{POH}$

PNH

PMH

Allergies

Dnugs

Eye.drops

OE

Ext. Eye

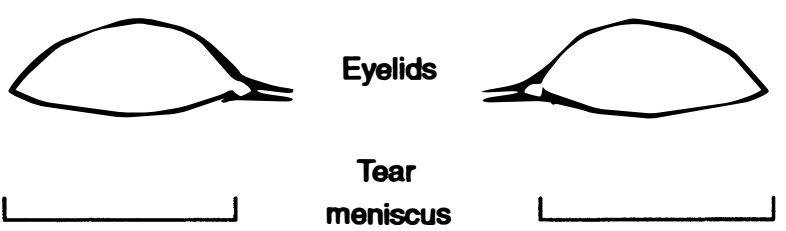

F D R T

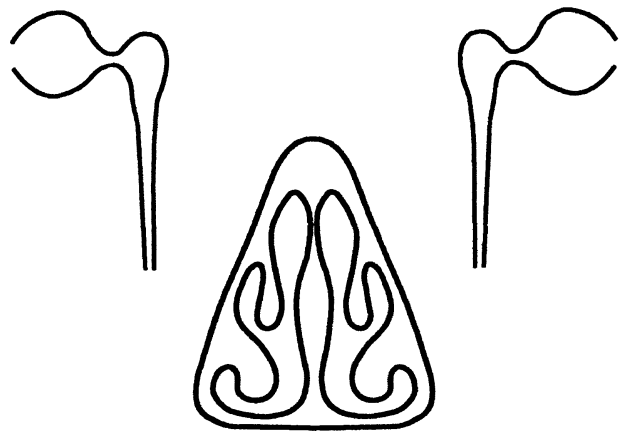

Findings

P.lan

\section{Doctor}

Fig. 1. Suggested lacrimal proforma.

\section{Findings on syringing}

Syringing findings are recorded using a line template for the canaliculi, sac and duct, with designated symbols indicating which canaliculus was irrigated, the direction of flow and any obstruction noted. An arrow over the lower canaliculus indicates that probing and irrigation was via that canaliculus; similarly an arrow over the upper canaliculus indicates that probing and irrigation was via the upper canaliculus. Regurgitation via one or both canaliculi is shown by a reversed arrow. If the regurgitation is slight and some fluid reaches the nose (suggestive of functional nasolacrimal duct stenosis) both sets of arrows may be broken, or only one arrow broken depending on the proportion of saline returning versus that descending the nasolacrimal duct. Regurgitation of mucus is noted. A palpable mucocoele is shown as a 'fat' sac. 
Table 1. Symbols used for puncta findings

\begin{tabular}{|l|l|l|}
\hline Normal punctum & Small filled circle & \\
\hline Visible but occluded punctum & Cross over small filled circle & $\times$ \\
\hline No evidence of punctum & Cross over expected location & $\times$ \\
\hline Stenosed punctum & Tiny dot surrounded by circle & $\odot$ \\
\hline Enlarged punctum, e.g. after 3 snip & Filled triangle on its side & \\
\hline Punctal medialisation & Small arrow pointing medially & $\rightarrow$ \\
\hline Punctal plug & Solid T & 4 \\
\hline
\end{tabular}

Complete common canalicular block is represented by a continuous vertical line and a partial (membranous) block by a broken vertical line. Individual canalicular findings are similarly recorded. The length of patent canaliculus is recorded in millimetres. If the canaliculus cannot be probed, it is represented by a dotted line.

\section{Combination of syringing and endoscopic ostium dye test after surgery}

Syringing findings after dacryocystorhinostomy (DCR) are recorded on a different line template together with the ostium findings from nasal endoscopy. The line template includes the canaliculi, sac and only the upper part of the nasolacrimal duct. The ostium is shown as an opening in the medial wall of the sac. The canaliculus is probed and irrigated and any regurgitation of saline shown by arrows. An arrow passing through the ostium from the sac into the nose is used if the syringing was patent and the functional endoscopic dye test (FEDT) was positive. A positive FEDT is recorded when a drop of fluorescein $2 \%$ placed in the conjunctival sac dilutes the tears and is seen emerging from the ostium. A thick line is placed over the ostium if it is found closed (fibrosis) and the FEDT marked -ve.

If the syringing is patent, the ostium closed and the FEDT negative, but dye is seen emerging from the lower end of the nasolacrimal duct endonasally, an arrow at the lower end of the duct indicates this. When the syringing is patent, the ostium closed and the FEDT negative but no dye is seen at the lower end of the nasolacrimal duct, an alternative drainage route is implied (perhaps through the ethmoids); 'alternative route' is written adjacent to the line drawing.

\section{Nasal endoscopic findings}

The findings from endoscopic endonasal examination with a rigid Hopkins endoscope $\left(0^{\circ}\right.$ or $\left.30^{\circ}\right)$ before and after surgery are recorded diagrammatically. A line template represent a coronal slice of the nasal space, at the level of the lacrimal sac. It includes the middle and inferior turbinates on the lateral nasal wall and the nasal septum centrally. The mucosal features are described in text. Anatomical and pathological findings are shown diagrammatically. A functioning ostium is shown by an open circle in the region of the lacrimal sac, usually at the anterior end of the middle turbinate. '+ve FEDT' can be written next to it confirming patency. If the surgical ostium has closed, this is written clearly in text and no circle is indicated.

\section{Results}

Diagrammatic representations of typical eyelid findings are shown in Fig. 2. An example recording the tear meniscus height with its slit lamp measure is shown in Fig. 3. Several examples of normal and abnormal syringing findings before lacrimal surgery are shown in Fig. 4. Examples of post-operative syringing findings combined with endoscopic ostium assessment are shown in Fig. 5. A variety of pre- and post-operative nasal findings from rigid endoscopy are shown in Fig. 6.

\section{Discussion}

This system of recording lacrimal findings provides a clear permanent record in the patient's notes which is useful when planning and monitoring surgery. The diagrams initially seem elaborate but are based on standard principles and symbols. These diagrams do not have to be formally memorised, only the underlying principles understood. We have included a standard proforma for use in the clinic; however, readers can create their own form using this diagrammatic method. It allows for both a systematic approach to lacrimal assessment before and after surgery as well as an easy interpretable system for recording the findings. We have chosen to develop diagrammatic representation because it is concise and enables the observer to record the clinical examination carefully. We have not provided a comprehensive account on how to take a lacrimal history, examine and investigate a patient with epiphora as this is available from current textbooks.

A precedent exists in other subspecialties of ophthalmology for recording the clinical examination diagrammatically. ${ }^{10-14}$ In particular, the simple system designed by Bron ${ }^{13}$ for documenting corneal disease is in general use and the system developed by Morris and Vivian $^{11}$ (based on Scott, Iowa) for strabismus is also well established. An expert group was required to develop the current diagrammatic system for grading and recording the fundal appearance of retinopathy of prematurity, which takes into account prognosis and progression of disease. The system we describe for lacrimal disease has its origin in the simple line drawings 

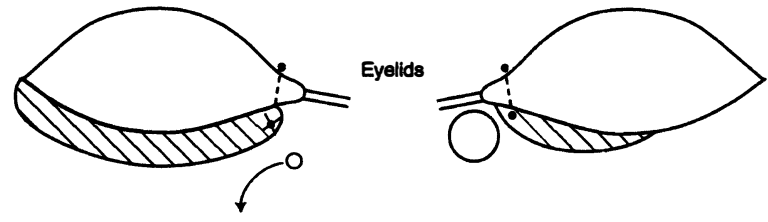

Fig. 2. Diagrammatic representation of eyelids, puncta and medial canthi. On the right there is a complete ectropion with secondary occluded lower punctum and a normal upper punctum. A fistula is draining from the region of the lacrimal sac. On the left there is a medial lower lid ectropion and palpable lacrimal sac mucocoele. Note that the lacrimal sac is predominantly below the level of the medial canthal tendon. A circle above the tendon would indicate different pathology, for instance a mucocoele arising from the ethmoid sinus. i)

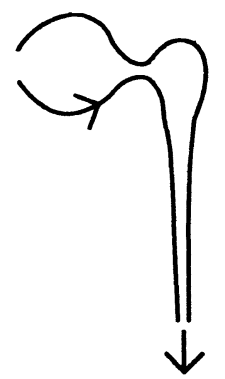

ii)

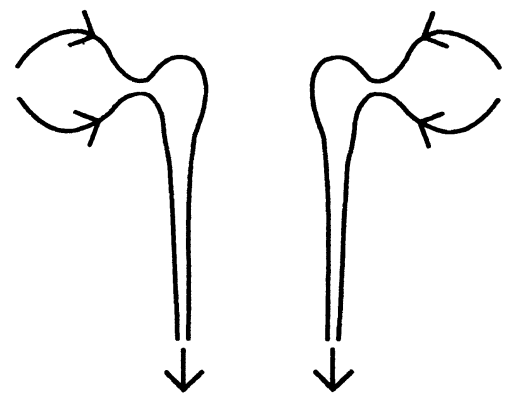

iii)

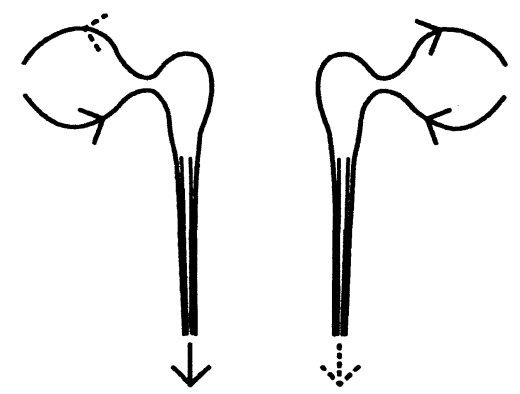

iv)

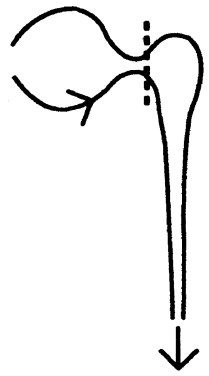

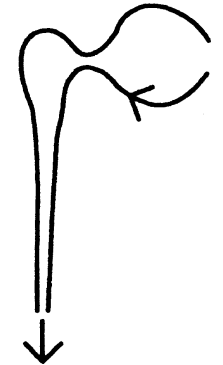

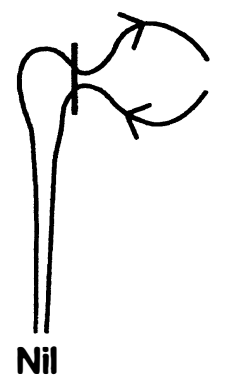

v)<smiles>C1C2CC3CC1CC(C2)C3</smiles>
tear meniscus normal limits, left is elevated.
Fig. 3. Recording of tear meniscus heights based on slit-lamp measurements prior to the instillation of any drops. Right is within vi)<smiles>CC1CCCCC12CCCCC2</smiles>

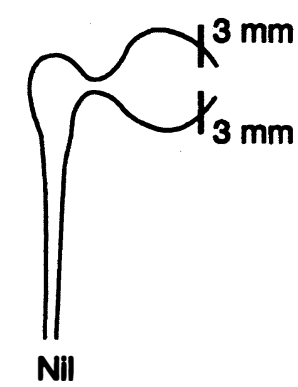

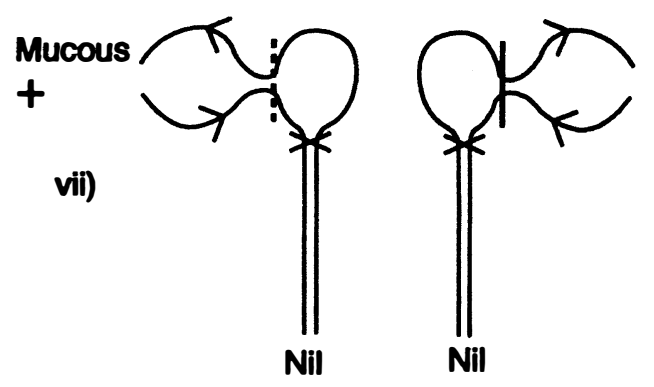

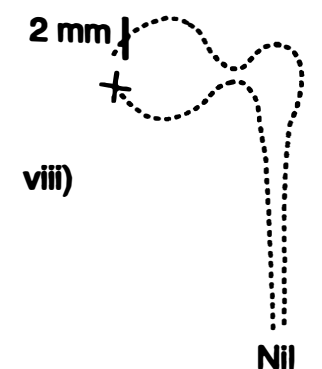<smiles>CC12CC3CC(C1)CC(C)(C3)C2</smiles>

Fig. 4. (i) Bilateral normal syringing and probing into the sac performed via the lower canaliculus. (ii) Bilateral normal syringing and probing into the sac performed via both lower and upper canaliculus. (iii) Functional epiphora from nasolacrimal duct stenosis - worse on the left. (iv) Right partial and left complete distal common canalicular block. (v) Right complete upper and lower canalicular block. Left complete proximal common canalicular block. (vi) Right absent lower punctum - patent syringing via upper canaliculus. Left complete proximal upper and lower canaliculi block. (vii) Right expressible mucocoele. Left non-expressible mucocoele. (viii) Right upper canalicular proximal block and absent lower punctum. Left partial distal upper canalicular block. 


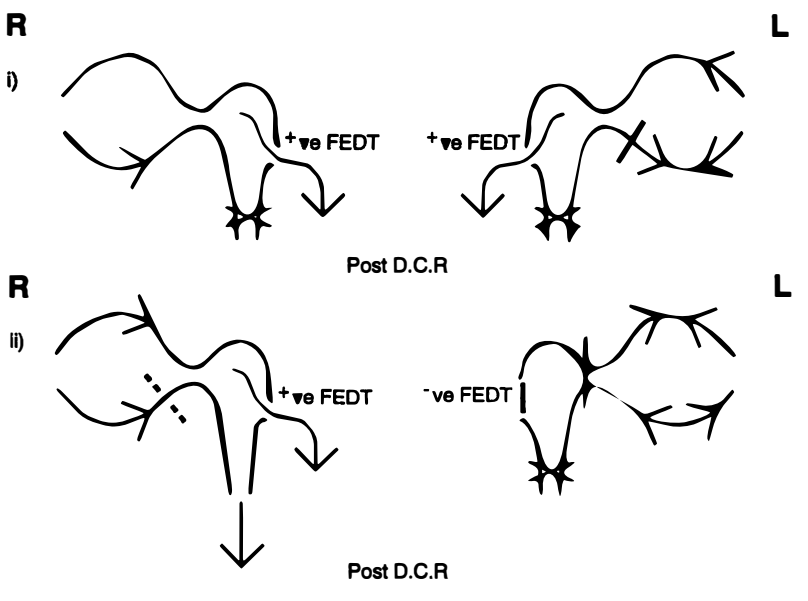

Fig. 5. Diagrammatic record of post-dacryocystorhinostomy (D.C.R.) syringing and ostium visualisation. (i) Right ostium patent via lower canaliculus. Left ostium patent via upper canaliculus. Distal block lower canaliculus. Bilateral +ve FEDT. (ii) Right partial block distal lower canaliculus, patent upper canaliculus. Ostium and nasolacrimal duct patent. The latter is seen on endoscopy as fluorescein emerging from the nasolacrimal duct opening in the inferior meatus and settling on the nasal floor. +ve FEDT. Left distal common canalicular block. Ostium closed. -ve FEDT.

everyone uses in the patient's notes and has been described by Hurwitz. ${ }^{15}$ Our system differs from the approach by Jordan ${ }^{16}$ who emphasises the findings from the Jones I and II tests.

Many of the traditional lacrimal tests, Jones tests, saccharine test and FDRT have variable results and are unreliable if used in isolation. ${ }^{1,5-7}$ For example, in the normal population the Jones I test has been shown to be negative with no dye in the inferior meatus despite a patent system in $22 \%{ }^{5}$ and $48 \%{ }^{6}$ of subjects. It is important that a diagrammatic record of the lacrimal examination reflects current lacrimal tests, including nasal endoscopy which has emerged as a crucial investigative instrument in the lacrimal examination. ${ }^{9}$ It not only allows visualisation of the normal anatomy but may be used to visualise ostium function by observing the FEDT, which is a modification of the Jones $\mathrm{I}_{\mathrm{E}}$ test. ${ }^{9}$

A diagrammatic system for recording the lacrimal findings must include the lids and periorbital area since conditions such as ectropion are a significant and treatable cause of epiphora and blepharitis a cause of hypersecretion.

Many ophthalmologists find the diagnosis of functional epiphora difficult to confirm. In the absence of a lid cause (including facial nerve weakness or paralysis) functional epiphora is usually due to progressive stenosis of the nasolacrimal duct from early primary acquired nasolacrimal duct obstruction (PANDO). This is characterised by chronic inflammation of the lacrimal duct mucosa with progressive fibrosis and narrowing. Our system of assessment and recording of findings would show an increased tear meniscus height, positive FDRT, some resistance to gentle syringing with regurgitation of saline as well as flow to the throat. The clinical diagnosis is confirmed by nuclear lacrimal scintillography.
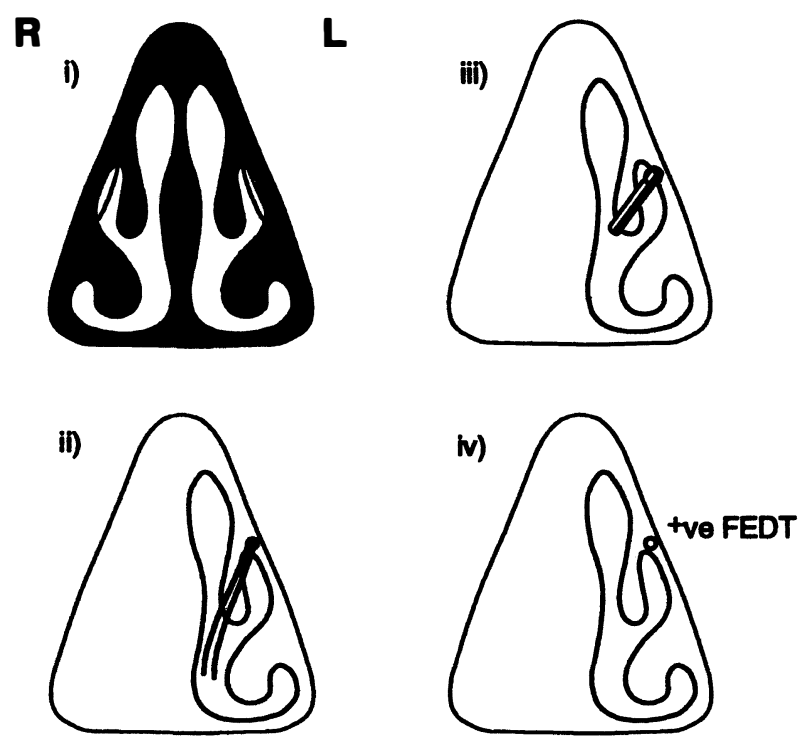

Fig. 6. Diagrammatic record of endonasal endoscopic findings. (i) Normal nasal anatomy. (ii) Left silicone O'Donoghue tubes in situ. (iii) Left functioning Jones tube. (iv) Left functioning DCR ostium.

The use of adjunctive investigations such as digital subtraction dacryocystography, CT or MRI scanning or nuclear lacrimal scintillography, assists in assessment of the lacrimal system and for planning surgery. The results of these ancillary tests are not recorded diagrammatically since they are available for inspection.

Transpunctal micro-endoscopic visualisation of the canalicular lumen is emerging as a potentially useful method of directly assessing and treating pathology, and this diagrammatic system would have to be expanded to take into account specific intraluminal findings.

We propose the described diagrammatic method for recording the lacrimal examination for general use. Recording clinical findings diagrammatically is not a new concept. This modified method is based on existing concepts and provides a clear documentation when more than one diagnostic test is required and the patient is seen by several different ophthalmologists. The principles are easy to learn and interpret since they include diagrams with shortened notation.

\section{References}

1. Conway ST. Evaluation and management of 'functional' nasolacrimal blockage: results of a survey of the American Society of Ophthalmic Plastic and Reconstructive surgery. Ophthalmic Plast Reconstr Surg 1994;10:185-7.

2. Schirmer O. Studien zur Physiologie und Pathologie der Tränenabsonderung und Tränenabfuhr. Arch Ophthalmol 1903;56:197.

3. Jones LT, Linn ML. The diagnosis and causes of epiphora. Am J Ophthalmol 1969;67:751.

4. Hornblass A. A simple taste test for lacrimal function. Arch Ophthalmol 1973;90:435.

5. Zappia RJ, Milder B. Lacrimal drainage function. 1. The Jones fluorescein test. 2 . The fluorescein dye disappearance test. Am J Ophthalmol 1972;74:154 62. 
6. Hornblass A, Ingis TM. Lacrimal function tests. Arch Ophthalmol 1979;97:1654-5.

7. Guzek JP, Ching AS, Hoang TA, et al. Clinical and radiologic lacrimal testing in patients with epiphora. Ophthalmology 1997;104:1875-81.

8. Welham RAN. Investigations for patients undergoing lacrimal surgery. Eye 1998;12:334-6.

9. Enzer YR, Shorr N. The Jones IE test: cobalt blue endoscopic primary dye test of lacrimal excretory function. Ophthalmic Plast Reconstr Surg 1997;13:204-9.

10. Doughty DD, Lennarson LW, Scott WE. A graphic portrayal of versions. Perspect Ophthalmol 1978;2:55-9.

11. Vivian AJ, Morris RJ. Diagrammatic representation of strabismus. Eye 1993;7:565-71.
12. Kaye SB, O'Donnell N, Holden R. Recording sensory and motor aspects of strabismus. J Pediatr Ophthalmol Strabismus 1997;34:188-90.

13. Bron AJ. A simple scheme for documenting corneal disease. Br J Ophthalmol 1973;57:629-34.

14. Sparrow JM, Bron AJ, Brown NAP, Ayliffe W, Hill AR. The Oxford Clinical Cataract Classification and Grading System. Int Ophthalmol 1986;9:207-25.

15. Hurwitz JJ. In: Hurwitz JJ, ed. The lacrimal system. 1st ed. Philadelphia: Lippincott-Raven, 1996:47-56.

16. Jordan DJ. Evaluation of the tearing patient. In: Bosniak S, editor. Principles and practice of ophthalmic plastic and reconstructive surgery. 1st ed. Philadelphia: Saunders, 1996:748-61. 